\title{
Convergence of Common Fixed Point for Asymptotically Quasi-Nonexpansive Mappings in Convex Metric Spaces
}

\author{
Gurucharan Singh Saluja and Hemant Kumar Nashine
}

\begin{abstract}
In this paper, the necessary and sufficient conditions for three-step iterative sequences with errors to converge to a common fixed point for three asymptotically quasi-nonexpansive mappings is established in convex metric spaces. The results of this paper are generalizations and improvements of the corresponding results of Chang [1] - [3], Kim et al. [8], Liu [9] - [11], Ghosh and Debnath [4], Xu and Noor [15], Shahzad and Udomene [13], Khan and Takahashi [6] and Khan and Ud-din [7].
\end{abstract}

\section{Introduction And Preliminaries}

Throughout this paper, we assume that $E$ is a metric space, $F(T)$ and $D(T)$ are the set of fixed points and domain of $T$ respectively and $\mathbb{N}$ is the set of all positive integers.

Definition 1.1 ( [8]). Let $T: D(T) \subset E \rightarrow E$ be a mapping.

(1) The mapping $T$ is said to be nonexpansive if

$$
d(T x, T y) \leq d(x, y), \quad \forall x, y \in D(T) .
$$

(2) The mapping $T$ is said to be quasi-nonexpansive if

$$
d(T x, p) \leq d(x, p), \quad \forall x \in D(T), \forall p \in F(T) .
$$

(3) The mapping $T$ is said to be asymptotically nonexpansive if there exists a sequence $r_{n} \in[0, \infty)$ with $\lim _{n \rightarrow \infty} r_{n}=0$ such that

$$
d\left(T^{n} x, T^{n} y\right) \leq\left(1+r_{n}\right) d(x, y), \quad \forall x, y \in D(T), \forall n \in \mathbb{N} .
$$

(4) The mapping $T$ is said to be asymptotically quasi-nonexpansive if there exists a sequence $r_{n} \in[0, \infty)$ with $\lim _{n \rightarrow \infty} r_{n}=0$ such that

$$
d\left(T^{n} x, p\right) \leq\left(1+r_{n}\right) d(x, p), \quad \forall x \in D(T), \forall p \in F(T), \forall n \in \mathbb{N} .
$$

2000 Mathematics Subject Classification. Primary: 41A50; Secondary: 41A65, 47H10.

Key words and phrases. Convex metric space, asymptotically quasi-nonexpansive mapping, asymptotically nonexpansive mapping, three-step iterative sequence with errors, common fixed point. 
Remark 1.1. From the definition 1.1, it follows that if $F(T)$ is nonempty, then a nonexpansive mapping is quasi-nonexpansive, and an asymptotically nonexpansive mapping is asymptotically quasi-nonexpansive. But the converse does not hold.

The iterative approximation problems of fixed points for asymptotically nonexpansive mappings or asymptotically quasi-nonexpansive mappings in Hilbert spaces or Banach spaces have been studied extensively by many others. In 1973, Petryshyn and Williamson [12] obtained a necessary and sufficient condition for Picard iterative sequences and Mann iterative sequences to converge to a fixed point for quasi-nonexpansive mappings and later, the result of [12] was extended by Ghosh and Debnath [4] to Ishikawa iterative sequences. Recently, Chang [1] - [3] has proved some other kinds of necessary and sufficient conditions for Ishikawa iterative sequences with errors to converge to a fixed point for asymptotically nonexpansive mappings and $\mathrm{Xu}$ and Noor [15] have established a convergence theorem of three-step iterative sequences with errors for asymptotically nonexpansive mappings in uniformly convex Banach spaces. In particular, Liu [9] obtained a necessary and sufficient condition for Ishikawa iterative sequences of asymptotically quasi-nonexpansive mappings in Banach spaces to converge to a fixed point and he [10] has also extended his result [9] to Ishikawa iterative sequences with errors. Furthermore, Kim et al. [8] extended the result of Liu [9] to modified three-step iterative sequences with mixed errors.

In 2001, Khan and Takahashi [6] have established a convergence theorem for two asymptotically nonexpansive mappings and in 2004, Khan and Ud-din [7] have established a convergence theorem for a scheme with errors for two nonexpansive mappings. In 2006, Shahzad and Udomene [13] extended the corresponding result of [6] and [7] for two asymptotically quasinonexpansive mappings and also established a necessary and sufficient condition for convergence of two asymptotically quasi-nonexpansive mappings in Banach spaces.

The purpose of this paper is to study some necessary and sufficient conditions for three-step iterative sequences with errors to converge to common fixed points for three asymptotically quasi-nonexpansive mappings in convex metric spaces. The results of this paper are generalization and improvements of the corresponding results in Chang [1] - [3], Ghosh and Debnath [4], Uddin and Khan [5], Khan and Takahashi [6], Khan and Ud-din [7], Kim et al. [8], Liu [9] - [11], Shahzad and Udomene [13] and Xu and Noor [15].

For the sake of convenience, we first recall some definitions and notations.

Definition 1.2. Let $(E, d)$ be a metric space and $I=[0,1]$. A mapping $W: E^{3} \times I^{3} \rightarrow E$ is said to be a convex structure on $E$ if it satisfies the following conditions: for all $u, x, y, z \in E$ and for all $\alpha, \beta, \gamma \in I$ with $\alpha+$ $\beta+\gamma=1$, 
(1) $W(x, y, z ; \alpha, 0,0)=x$,

(2) $d(u, W(x, y, z ; \alpha, \beta, \gamma)) \leq \alpha d(u, x)+\beta d(u, y)+\gamma d(u, z)$.

If $(E, d)$ is a metric space with a convex structure $W$, then $(E, d)$ is called a convex metric space and denotes it by $(E, d, W)$.

Remark 1.2. Every linear normed space is a convex metric space, where a convex structure $W(x, y, z ; \alpha, \beta, \gamma)=\alpha x+\beta y+\gamma z$, for all $x, y, z \in E$ and $\alpha, \beta, \gamma \in I$ with $\alpha+\beta+\gamma=1$. But there exist some convex metric spaces which can not be embedded into any linear normed spaces (see, Takahashi [14]).

Definition 1.3. (1) Let $(E, d, W)$ be a convex metric space, $T_{1}, T_{2}, T_{3}: E \rightarrow$ $E$ be mappings and let $x_{1} \in E$ be a given point. Then the sequence $\left\{x_{n}\right\}$ defined by

$$
\begin{aligned}
x_{n+1} & =W\left(x_{n}, T_{1}^{n} y_{n}, u_{n} ; a_{n}, b_{n}, c_{n}\right), \\
y_{n} & =W\left(x_{n}, T_{2}^{n} z_{n}, v_{n} ; a_{n}^{\prime}, b_{n}^{\prime}, c_{n}^{\prime}\right), \\
z_{n} & =W\left(x_{n}, T_{3}^{n} x_{n}, w_{n} ; a_{n}^{\prime \prime}, b_{n}^{\prime \prime}, c_{n}^{\prime \prime}\right), \quad \forall n \in \mathbb{N},
\end{aligned}
$$

is called the three-step iterative sequence with errors for three mappings $T_{1}, T_{2}, T_{3}$, where $\left\{a_{n}\right\},\left\{a_{n}^{\prime}\right\},\left\{a_{n}^{\prime \prime}\right\},\left\{b_{n}\right\},\left\{b_{n}^{\prime}\right\},\left\{b_{n}^{\prime \prime}\right\},\left\{c_{n}\right\},\left\{c_{n}^{\prime}\right\}$ and $\left\{c_{n}^{\prime \prime}\right\}$ are nine sequences in $[0,1]$ satisfying the following conditions:

$$
a_{n}+b_{n}+c_{n}=a_{n}^{\prime}+b_{n}^{\prime}+c_{n}^{\prime}=a_{n}^{\prime \prime}+b_{n}^{\prime \prime}+c_{n}^{\prime \prime}=1, \forall n \in \mathbb{N} \text {, }
$$

and $\left\{u_{n}\right\},\left\{v_{n}\right\},\left\{w_{n}\right\}$ are three bounded sequences in $E$.

(2) In (1.1), if $b_{n}^{\prime \prime}=c_{n}^{\prime \prime}=0$, for all $n=1,2, \ldots$, then $z_{n}=x_{n}$ and $T_{1}=T_{2}=T_{3}=T$. Then the sequence $\left\{x_{n}\right\}$ defined by

$$
\begin{aligned}
x_{n+1} & =W\left(x_{n}, T^{n} y_{n}, u_{n} ; a_{n}, b_{n}, c_{n}\right), \\
y_{n} & =W\left(x_{n}, T^{n} x_{n}, v_{n} ; a_{n}^{\prime}, b_{n}^{\prime}, c_{n}^{\prime}\right), \quad \forall n \in \mathbb{N},
\end{aligned}
$$

is called the Ishikawa type (or two-step) iterative sequence with errors for the mapping $T$, where $\left\{a_{n}\right\},\left\{b_{n}\right\},\left\{c_{n}\right\},\left\{a_{n}^{\prime}\right\},\left\{b_{n}^{\prime}\right\}$ and $\left\{c_{n}^{\prime}\right\}$ are six sequences in $[0,1]$ satisfying the conditions:

$a_{n}+b_{n}+c_{n}=a_{n}^{\prime}+b_{n}^{\prime}+c_{n}^{\prime}=1, \forall n \in \mathbb{N}$,

and $\left\{u_{n}\right\},\left\{v_{n}\right\}$ are two bounded sequences in $E$.

\section{Main Results}

In order to prove our main result, we will first prove the following lemma.

Lemma 2.1. Let $(E, d, W)$ be a convex metric space, $T_{1}, T_{2}, T_{3}: E \rightarrow E$ be three asymptotically quasi-nonexpansive mappings satisfying $\sum_{n=1}^{\infty} r_{n}<$ $\infty$ where $\left\{r_{n}\right\}$ is the sequence appeared in Definition 1.1, and $F=\bigcap_{i=1}^{3} F\left(T_{i}\right)$ be a nonempty set. For a given $x_{1} \in E$, let $\left\{x_{n}\right\}$ be the three-step iterative sequences with errors defined by (1.1). Then 
(a) $d\left(x_{n+1}, p\right) \leq\left(1+r_{n}\right)^{3} d\left(x_{n}, p\right)+B_{n}, \forall p \in F, n \in \mathbb{N}$, where $B_{n}=A_{n} b_{n}\left(1+r_{n}\right)+c_{n} d\left(u_{n}, p\right), A_{n}=b_{n}^{\prime} c_{n}^{\prime \prime}\left(1+r_{n}\right) d\left(w_{n}, p\right)+$ $c_{n}^{\prime} d\left(v_{n}, p\right)$

and $\left\{u_{n}\right\},\left\{v_{n}\right\},\left\{w_{n}\right\}$ are three bounded sequences in $E$.

(b) there exists a constant $M>0$ such that

$$
d\left(x_{m}, p\right) \leq M \cdot d\left(x_{n}, p\right)+M \cdot \sum_{j=n}^{m-1} B_{j}, \forall p \in F, m>n .
$$

Proof. (a) Let $p \in F=\bigcap_{i=1}^{3} F\left(T_{i}\right)$. Since $T_{i}(i=1,2,3)$ is asymptotically quasi-nonexpansive, we have

$$
\begin{aligned}
d\left(x_{n+1}, p\right) & =d\left(W\left(x_{n}, T_{1}^{n} y_{n}, u_{n} ; a_{n}, b_{n}, c_{n}\right), p\right) \\
& \leq a_{n} d\left(x_{n}, p\right)+b_{n} d\left(T_{1}^{n} y_{n}, p\right)+c_{n} d\left(u_{n}, p\right) \\
& \leq a_{n} d\left(x_{n}, p\right)+b_{n}\left(1+r_{n}\right) d\left(y_{n}, p\right)+c_{n} d\left(u_{n}, p\right)
\end{aligned}
$$

$$
\begin{aligned}
d\left(y_{n}, p\right) & =d\left(W\left(x_{n}, T_{2}^{n} z_{n}, v_{n} ; a_{n}^{\prime}, b_{n}^{\prime}, c_{n}^{\prime}\right), p\right) \\
& \leq a_{n}^{\prime} d\left(x_{n}, p\right)+b_{n}^{\prime} d\left(T_{2}^{n} z_{n}, p\right)+c_{n}^{\prime} d\left(v_{n}, p\right) \\
& \leq a_{n}^{\prime} d\left(x_{n}, p\right)+b_{n}^{\prime}\left(1+r_{n}\right) d\left(z_{n}, p\right)+c_{n}^{\prime} d\left(v_{n}, p\right)
\end{aligned}
$$

and

$$
\begin{aligned}
d\left(z_{n}, p\right) & =d\left(W\left(x_{n}, T_{3}^{n} x_{n}, w_{n} ; a_{n}^{\prime \prime}, b_{n}^{\prime \prime}, c_{n}^{\prime \prime}\right), p\right) \\
& \leq a_{n}^{\prime \prime} d\left(x_{n}, p\right)+b_{n}^{\prime \prime} d\left(T_{3}^{n} x_{n}, p\right)+c_{n}^{\prime \prime} d\left(w_{n}, p\right) \\
& \leq a_{n}^{\prime \prime} d\left(x_{n}, p\right)+b_{n}^{\prime \prime}\left(1+r_{n}\right) d\left(x_{n}, p\right)+c_{n}^{\prime \prime} d\left(w_{n}, p\right) \\
& \leq a_{n}^{\prime \prime}\left(1+r_{n}\right) d\left(x_{n}, p\right)+b_{n}^{\prime \prime}\left(1+r_{n}\right) d\left(x_{n}, p\right)+c_{n}^{\prime \prime} d\left(w_{n}, p\right) \\
& \leq\left(a_{n}^{\prime \prime}+b_{n}^{\prime \prime}\right)\left(1+r_{n}\right) d\left(x_{n}, p\right)+c_{n}^{\prime \prime} d\left(w_{n}, p\right) \\
& =\left(1-c_{n}^{\prime \prime}\right)\left(1+r_{n}\right) d\left(x_{n}, p\right)+c_{n}^{\prime \prime} d\left(w_{n}, p\right) \\
& \leq\left(1+r_{n}\right) d\left(x_{n}, p\right)+c_{n}^{\prime \prime} d\left(w_{n}, p\right)
\end{aligned}
$$


Substituting (2.3) into (2.2), we have

$$
\begin{aligned}
d\left(y_{n}, p\right) \leq & a_{n}^{\prime} d\left(x_{n}, p\right)+b_{n}^{\prime}\left(1+r_{n}\right)\left[\left(1+r_{n}\right) d\left(x_{n}, p\right)+c_{n}^{\prime \prime} d\left(w_{n}, p\right)\right] \\
& +c_{n}^{\prime} d\left(v_{n}, p\right) \\
\leq & a_{n}^{\prime} d\left(x_{n}, p\right)+b_{n}^{\prime}\left(1+r_{n}\right)^{2} d\left(x_{n}, p\right)+b_{n}^{\prime}\left(1+r_{n}\right) c_{n}^{\prime \prime} d\left(w_{n}, p\right) \\
& +c_{n}^{\prime} d\left(v_{n}, p\right) \\
\leq & a_{n}^{\prime} d\left(x_{n}, p\right)+b_{n}^{\prime}\left(1+r_{n}\right)^{2} d\left(x_{n}, p\right)+b_{n}^{\prime}\left(1+r_{n}\right) c_{n}^{\prime \prime} d\left(w_{n}, p\right) \\
& +c_{n}^{\prime} d\left(v_{n}, p\right) \\
\leq & a_{n}^{\prime}\left(1+r_{n}\right)^{2} d\left(x_{n}, p\right)+b_{n}^{\prime}\left(1+r_{n}\right)^{2} d\left(x_{n}, p\right)+b_{n}^{\prime}\left(1+r_{n}\right) c_{n}^{\prime \prime} d\left(w_{n}, p\right) \\
& +c_{n}^{\prime} d\left(v_{n}, p\right) \\
\leq & \left(a_{n}^{\prime}+b_{n}^{\prime}\right)\left(1+r_{n}\right)^{2} d\left(x_{n}, p\right)+b_{n}^{\prime}\left(1+r_{n}\right) c_{n}^{\prime \prime} d\left(w_{n}, p\right)+c_{n}^{\prime} d\left(v_{n}, p\right) \\
= & \left(1-c_{n}^{\prime}\right)\left(1+r_{n}\right)^{2} d\left(x_{n}, p\right)+b_{n}^{\prime}\left(1+r_{n}\right) c_{n}^{\prime \prime} d\left(w_{n}, p\right)+c_{n}^{\prime} d\left(v_{n}, p\right) \\
\leq & \left(1+r_{n}\right)^{2} d\left(x_{n}, p\right)+A_{n}
\end{aligned}
$$

where $A_{n}=b_{n}^{\prime}\left(1+r_{n}\right) c_{n}^{\prime \prime} d\left(w_{n}, p\right)+c_{n}^{\prime} d\left(v_{n}, p\right)$. And again, substituting (2.4) into (2.1), it follows that

$$
\begin{aligned}
d\left(x_{n+1}, p\right) \leq & a_{n} d\left(x_{n}, p\right)+b_{n}\left(1+r_{n}\right)\left[\left(1+r_{n}\right)^{2} d\left(x_{n}, p\right)+A_{n}\right] \\
& +c_{n} d\left(u_{n}, p\right) \\
\leq & a_{n} d\left(x_{n}, p\right)+b_{n}\left(1+r_{n}\right)^{3} d\left(x_{n}, p\right)+b_{n}\left(1+r_{n}\right) A_{n}+c_{n} d\left(u_{n}, p\right) \\
\leq & a_{n}\left(1+r_{n}\right)^{3} d\left(x_{n}, p\right)+b_{n}\left(1+r_{n}\right)^{3} d\left(x_{n}, p\right)+b_{n}\left(1+r_{n}\right) A_{n}+c_{n} d\left(u_{n}, p\right) \\
\leq & \left(a_{n}+b_{n}\right)\left(1+r_{n}\right)^{3} d\left(x_{n}, p\right)+b_{n}\left(1+r_{n}\right) A_{n}+c_{n} d\left(u_{n}, p\right) \\
= & \left(1-c_{n}\right)\left(1+r_{n}\right)^{3} d\left(x_{n}, p\right)+b_{n}\left(1+r_{n}\right) A_{n}+c_{n} d\left(u_{n}, p\right) \\
\leq & \left(1+r_{n}\right)^{3} d\left(x_{n}, p\right)+B_{n}
\end{aligned}
$$

where $B_{n}=b_{n}\left(1+r_{n}\right) A_{n}+c_{n} d\left(u_{n}, p\right)$. This completes the proof of (a). 
(b) If $x \geq 0$, then $1+x \leq e^{x}$ and $(1+x)^{3} \leq e^{3 x}$. Therefore from (a) we can obtain that

$$
\begin{aligned}
d\left(x_{m}, p\right) & \leq\left(1+r_{m-1}\right)^{3} d\left(x_{m-1}, p\right)+B_{m-1} \\
& \leq e^{3 r_{m-1}} d\left(x_{m-1}, p\right)+B_{m-1} \\
& \leq e^{3 r_{m-1}}\left[e^{3 r_{m-2}} d\left(x_{m-2}, p\right)+B_{m-2}\right]+B_{m-1} \\
& \leq e^{3\left(r_{m-1}+r_{m-2}\right)} d\left(x_{m-2}, p\right)+e^{3 r_{m-1}} B_{m-2}+B_{m-1} \\
& \leq e^{3\left(r_{m-1}+r_{m-2}\right)} d\left(x_{m-2}, p\right)+e^{3 r_{m-1}}\left[B_{m-1}+B_{m-2}\right] \\
& \leq \ldots \ldots \\
& \leq \ldots \ldots \\
& \leq e^{3\left(r_{m-1}+r_{m-2}+\cdots+r_{n}\right)} d\left(x_{n}, p\right) \\
& +e^{3\left(r_{m-1}+r_{m-2}+\cdots+r_{n}\right)}\left[B_{m-1}+B_{m-2}+\cdots+B_{n}\right] \\
& \leq e^{3 \sum_{j=n}^{m-1} r_{j}} d\left(x_{n}, p\right)+e^{3 \sum_{j=n}^{m-1} r_{j}} \cdot \sum_{j=n}^{m-1} B_{j} \\
& \leq M . d\left(x_{n}, p\right)+M \cdot \sum_{j=n}^{m-1} B_{j},
\end{aligned}
$$

where $M=e^{3 \sum_{j=n}^{m-1} r_{j}}$. This completes the proof of (b).

Lemma 2.1 [10]. Let the number of sequences $\left\{a_{n}\right\},\left\{b_{n}\right\}$ and $\left\{\lambda_{n}\right\}$ satisfy that $a_{n} \geq 0, b_{n} \geq 0, \lambda_{n} \geq 0, a_{n+1} \leq\left(1+\lambda_{n}\right) a_{n}+b_{n}, \forall n \in \mathbb{N}$, $\sum_{n=1}^{\infty} b_{n}<\infty, \sum_{n=1}^{\infty} \lambda_{n}<\infty$. Then

(a) $\lim _{n \rightarrow \infty} a_{n}$ exists.

(b) If $\liminf _{n \rightarrow \infty} a_{n}=0$, then $\lim _{n \rightarrow \infty} a_{n}=0$.

Now, we are in a position to prove the main results. $D_{d}(y, S)$ denotes the distance from $y$ to set $S$, that is, $D_{d}(y, S)=\inf \{d(y, s): s \in S\}$.

Theorem 2.1. Let $(E, d, W)$ be a complete convex metric space, $T_{1}, T_{2}, T_{3}$ : $E \rightarrow E$ be three asymptotically quasi-nonexpansive mappings and $F=$ $\bigcap_{i=1}^{3} F\left(T_{i}\right)$ be a nonempty set. For a given $x_{1} \in E$, let $\left\{x_{n}\right\}$ be the threestep iterative sequence with errors defined by (1.1) and $\left\{r_{n}\right\},\left\{c_{n}\right\},\left\{c_{n}^{\prime}\right\}$, $\left\{c_{n}^{\prime \prime}\right\}$ be four sequences satisfying the following conditions:

(i) $\sum_{n=1}^{\infty} r_{n}<\infty$,

(ii) $\sum_{n=1}^{\infty} c_{n}<\infty, \sum_{n=1}^{\infty} c_{n}^{\prime}<\infty, \sum_{n=1}^{\infty} c_{n}^{\prime \prime}<\infty$,

where $\left\{r_{n}\right\}$ is a sequence appeared in Definition 1.1 and $\left\{c_{n}\right\},\left\{c_{n}^{\prime}\right\},\left\{c_{n}^{\prime \prime}\right\}$ are three sequences appeared in (1.1). Then the iterative sequence $\left\{x_{n}\right\}$ converges to a common fixed point of $\left\{T_{i}: i=1,2,3\right\}$ if and only if

$$
\liminf _{n \rightarrow \infty} D_{d}\left(x_{n}, F\right)=0 .
$$

Proof. The necessity is obvious. Now, we prove the sufficiency. Suppose that the condition $\liminf _{n \rightarrow \infty} D_{d}\left(x_{n}, F\right)=0$ is satisfied. Then from Lemma 
2.1(a), we have

$$
d\left(x_{n+1}, p\right) \leq\left(1+r_{n}\right)^{3} d\left(x_{n}, p\right)+B_{n}, \quad \forall p \in F, \quad \forall n \in \mathbb{N},
$$

where $B_{n}=b_{n}\left(1+r_{n}\right) A_{n}+c_{n} d\left(u_{n}, p\right)$ and $A_{n}=b_{n}^{\prime}\left(1+r_{n}\right) c_{n}^{\prime \prime} d\left(w_{n}, p\right)+$ $c_{n}^{\prime} d\left(v_{n}, p\right)$. Since $0 \leq b_{n}, b_{n}^{\prime} \leq 1, \sum_{n=1}^{\infty} r_{n}<\infty, \sum_{n=1}^{\infty} c_{n}<\infty, \sum_{n=1}^{\infty} c_{n}^{\prime}<$ $\infty, \sum_{n=1}^{\infty} c_{n}^{\prime \prime}<\infty$ and $\left\{u_{n}\right\},\left\{v_{n}\right\},\left\{w_{n}\right\}$ are three bounded sequences, we have $\sum_{n=1}^{\infty} A_{n}<\infty$ and so $\sum_{n=1}^{\infty} B_{n}<\infty$. From (2.5) we can obtain that

$$
D_{d}\left(x_{n+1}, F\right) \leq\left(1+r_{n}\right)^{3} D_{d}\left(x_{n}, F\right)+B_{n} .
$$

Since $\liminf \operatorname{in}_{n \rightarrow \infty} D_{d}\left(x_{n}, F\right)=0$, by Lemma 2.2 , we have

$$
\lim _{n \rightarrow \infty} D_{d}\left(x_{n}, F\right)=0 .
$$

Now, we will prove that $\left\{x_{n}\right\}$ is a Cauchy sequence. Let $\varepsilon>0$. By Lemma 2.1(b), there exists a constant $M>0$ such that

$$
d\left(x_{m}, p\right) \leq M \cdot d\left(x_{n}, p\right)+M \cdot \sum_{j=n}^{m-1} B_{j}, \forall p \in F, m>n .
$$

Since $\lim _{n \rightarrow \infty} D_{d}\left(x_{n}, F\right)=0$ and $\sum_{n=1}^{\infty} B_{n}<\infty$, there exists a constant $N_{1}$ such that for all $n \geq N_{1}$,

$$
D_{d}\left(x_{n}, F\right)<\frac{\varepsilon}{4 M} \text { and } \sum_{j=N_{1}}^{\infty} B_{j}<\frac{\varepsilon}{6 M} .
$$

We note that there exists $p_{1} \in F$ such that $d\left(x_{N_{1}}, p_{1}\right)<\frac{\varepsilon}{3 M}$. It follows that from (2.6) that for all $m>n>N_{1}$,

$$
\begin{aligned}
& d\left(x_{m}, x_{n}\right) \leq d\left(x_{m}, p_{1}\right)+d\left(x_{n}, p_{1}\right) \\
& \leq M \cdot d\left(x_{N_{1}}, p_{1}\right)+M \sum_{j=N_{1}}^{m-1} B_{j}+M d\left(x_{N_{1}}, p_{1}\right)+M \sum_{j=N_{1}}^{n-1} B_{j} \\
& <M \cdot \frac{\varepsilon}{3 M}+M \frac{\varepsilon}{6 M}+M \frac{\varepsilon}{3 M}+M \frac{\varepsilon}{6 M} \\
& =\varepsilon \text {. }
\end{aligned}
$$

Since $\varepsilon$ is an arbitrary positive number, (2.7) implies that $\left\{x_{n}\right\}$ is a Cauchy sequence. From the completeness of this, $\lim _{n \rightarrow \infty} x_{n}$ exists. Let $\lim _{n \rightarrow \infty} x_{n}=$ $p$. It will be proven that $p$ is a common fixed point. Let $\bar{\varepsilon}>0$. Since $\lim _{n \rightarrow \infty} x_{n}=p$, there exists a natural number $N_{2}$ such that for all $n \geq N_{2}$,

$$
d\left(x_{n}, p\right)<\frac{\bar{\varepsilon}}{2\left(2+r_{1}\right)} .
$$

$\lim _{n \rightarrow \infty} D_{d}\left(x_{n}, F\right)=0$ implies that there exists a natural number $N_{3} \geq N_{2}$ such that for all $n \geq N_{3}$,

$$
D_{d}\left(x_{n}, F\right)<\frac{\bar{\varepsilon}}{3\left(4+3 r_{1}\right)} \text {. }
$$


Therefore, there exists a $p^{*} \in F$ such that

$$
d\left(x_{N_{3}}, p^{*}\right)<\frac{\bar{\varepsilon}}{2\left(4+3 r_{1}\right)} .
$$

From (2.8) and (2.10), we have for any $i \in I$

$$
\begin{aligned}
d\left(T_{i} p, p\right) \leq & d\left(T_{i} p, p^{*}\right)+d\left(p^{*}, T_{i} x_{N_{3}}\right)+d\left(T_{i} x_{N_{3}}, p^{*}\right)+d\left(p^{*}, x_{N_{3}}\right)+d\left(x_{N_{3}}, p\right) \\
= & d\left(T_{i} p, p^{*}\right)+2 d\left(T_{i} x_{N_{3}}, p^{*}\right)+d\left(p^{*}, x_{N_{3}}\right)+d\left(x_{N_{3}}, p\right) \\
\leq & \left(1+r_{1}\right) d\left(p, p^{*}\right)+2\left(1+r_{1}\right) d\left(x_{N_{3}}, p^{*}\right)+d\left(p^{*}, x_{N_{3}}\right)+d\left(x_{N_{3}}, p\right) \\
\leq & \left(1+r_{1}\right)\left[d\left(p, x_{N_{3}}\right)+d\left(x_{N_{3}}, p^{*}\right)\right]+2\left(1+r_{1}\right) d\left(x_{N_{3}}, p^{*}\right) \\
& +d\left(p^{*}, x_{N_{3}}\right)+d\left(x_{N_{3}}, p\right) \\
= & \left(2+r_{1}\right) d\left(x_{N_{3}}, p\right)+\left(4+3 r_{1}\right) d\left(x_{N_{3}}, p^{*}\right) \\
< & \left(2+r_{1}\right) \cdot \frac{\bar{\varepsilon}}{2\left(2+r_{1}\right)}+\left(4+3 r_{1}\right) \cdot \frac{\bar{\varepsilon}}{2\left(4+3 r_{1}\right)} \\
= & \bar{\varepsilon} .
\end{aligned}
$$

Since $\bar{\varepsilon}$ is an arbitrary positive number, this implies that $T_{i} p=p$. Hence $p \in F\left(T_{i}\right)$ for all $i \in I$ and so $p \in F=\bigcap_{i=1}^{3} F\left(T_{i}\right)$. Thus the iterative sequence $\left\{x_{n}\right\}$ converges to a common fixed point of $\left\{T_{i}: i=1,2,3\right\}$. This completes the proof.

In (1.1), if $T_{1}=T_{2}=T_{3}=T, b_{n}^{\prime \prime}=c_{n}^{\prime \prime}=0$ for all $n=1,2, \ldots$, then $z_{n}=x_{n}$. Therefore, the following corollary can be obtained from Theorem 2.1 immediately.

Corollary 2.1. Let $(E, d, W)$ be a complete convex metric space, $T: E \rightarrow$ $E$ be an asymptotically quasi-nonexpansive mapping and $F(T)$ be a nonempty set. For a given $x_{1} \in E$, let $\left\{x_{n}\right\}$ be the Ishikawa type iterative sequence with errors defined by (1.2) and $\left\{r_{n}\right\},\left\{c_{n}\right\},\left\{c_{n}^{\prime}\right\}$ be three sequences satisfying the following conditions:

(i) $\sum_{n=1}^{\infty} r_{n}<\infty$,

(ii) $\sum_{n=1}^{\infty} c_{n}<\infty, \sum_{n=1}^{\infty} c_{n}^{\prime}<\infty$,

where $\left\{r_{n}\right\}$ is a sequence appeared in Definition 1.1 and $\left\{c_{n}\right\},\left\{c_{n}^{\prime}\right\}$ are two sequences appeared in (1.2). Then the iterative sequence $\left\{x_{n}\right\}$ converges to a fixed point of $T$ if and only if

$$
\liminf _{n \rightarrow \infty} D_{d}\left(x_{n}, F(T)\right)=0 .
$$

By using the same method in Theorem 2.1, we can easily obtain the following theorem.

Theorem 2.2. Let $(E, d, W)$ be a complete convex metric space, $T_{1}, T_{2}, T_{3}$ : $E \rightarrow E$ be three quasi-nonexpansive mappings and $F=\bigcap_{i=1}^{3} F\left(T_{i}\right)$ be a nonempty set. For a given $x_{1} \in E$, let $\left\{x_{n}\right\}$ be the three-step iterative 
sequence with errors defined by:

$$
\begin{aligned}
x_{n+1} & =W\left(x_{n}, T_{1} y_{n}, u_{n} ; a_{n}, b_{n}, c_{n}\right), \\
y_{n} & =W\left(x_{n}, T_{2} z_{n}, v_{n} ; a_{n}^{\prime}, b_{n}^{\prime}, c_{n}^{\prime}\right), \\
z_{n} & =W\left(x_{n}, T_{3} x_{n}, w_{n} ; a_{n}^{\prime \prime}, b_{n}^{\prime \prime}, c_{n}^{\prime \prime}\right), \quad \forall n \in \mathbb{N},
\end{aligned}
$$

and $\left\{c_{n}\right\},\left\{c_{n}^{\prime}\right\},\left\{c_{n}^{\prime \prime}\right\}$ are three sequences satisfying the following condition:

(i) $\sum_{n=1}^{\infty} c_{n}<\infty, \sum_{n=1}^{\infty} c_{n}^{\prime}<\infty, \sum_{n=1}^{\infty} c_{n}^{\prime \prime}<\infty$,

where $\left\{c_{n}\right\},\left\{c_{n}^{\prime}\right\},\left\{c_{n}^{\prime \prime}\right\}$ are three sequences appeared in (1.3). Then the iterative sequence $\left\{x_{n}\right\}$ converges to a common fixed point of $\left\{T_{i}: i=\right.$ $1,2,3\}$ if and only if

$$
\liminf _{n \rightarrow \infty} D_{d}\left(x_{n}, F\right)=0 .
$$

From Theorem 2.1, we can also obtain the following result for the Banach space.

Theorem 2.3. Let $E$ be a real Banach space, $T_{1}, T_{2}, T_{3}: E \rightarrow E$ be three asymptotically quasi-nonexpansive mappings satisfying the condition (i) in Theorem 2.1 and $F=\bigcap_{i=1}^{3} F\left(T_{i}\right)$ be a nonempty set. Let $\left\{x_{n}\right\}$ be the three-step iterative sequence with errors defined by

$$
\begin{gathered}
x_{1} \in E, \\
x_{n+1}=a_{n} x_{n}+b_{n} T_{1}^{n} y_{n}+c_{n} u_{n}, \\
y_{n}=a_{n}^{\prime} x_{n}+b_{n}^{\prime} T_{2}^{n} z_{n}+c_{n}^{\prime} v_{n}, \\
z_{n}=a_{n}^{\prime \prime} x_{n}+b_{n}^{\prime \prime} T_{3}^{n} x_{n}+c_{n}^{\prime \prime} w_{n}, \quad \forall n \in \mathbb{N},
\end{gathered}
$$

where $\left\{u_{n}\right\},\left\{v_{n}\right\},\left\{w_{n}\right\}$ are three bounded sequences in $E$ and $\left\{a_{n}\right\},\left\{a_{n}^{\prime}\right\}$, $\left\{a_{n}^{\prime \prime}\right\},\left\{b_{n}\right\},\left\{b_{n}^{\prime}\right\},\left\{b_{n}^{\prime \prime}\right\},\left\{c_{n}\right\},\left\{c_{n}^{\prime}\right\}$ and $\left\{c_{n}^{\prime \prime}\right\}$ are nine sequences in $[0,1]$ satisfying $a_{n}+b_{n}+c_{n}=a_{n}^{\prime}+b_{n}^{\prime}+c_{n}^{\prime}=a_{n}^{\prime \prime}+b_{n}^{\prime \prime}+c_{n}^{\prime \prime}=1, \forall n \in \mathbb{N}$, and $\sum_{n=1}^{\infty} c_{n}<\infty, \sum_{n=1}^{\infty} c_{n}^{\prime}<\infty, \sum_{n=1}^{\infty} c_{n}^{\prime \prime}<\infty$. Then the iterative sequence $\left\{x_{n}\right\}$ converges to a common fixed point of $\left\{T_{i}: i=1,2,3\right\}$ if and only if

$$
\liminf _{n \rightarrow \infty} D\left(x_{n}, F\right)=0 .
$$

where $D_{d}(y, S)=\inf \{d(y, s): s \in S\}$.

Proof. Since $E$ is a Banach space, it is a complete convex metric space with a convex structure $W(x, y, z: \alpha, \beta, \gamma):=\alpha x+\beta y+\gamma z$, for all $x, y, z \in E$ and for all $\alpha, \beta, \gamma \in[0,1]$ with $\alpha+\beta+\gamma=1$. Therefore, the conclusion of Theorem 2.3 can be obtained from Theorem 2.1 immediately.

Remark 2.1. (1) Theorem 2.1 and 2.2 are two new convergence theorems of three-step iterative sequences with errors for nonlinear mappings in convex metric spaces. These two theorems generalize and improves the corresponding results of [9]- [11], [1]- [3] and [4, 6, 7, 12, 13, 15]. 
(2) Theorem 2.3 generalizes and improves the corresponding results of Kim et al. [8], Liu [10, 11], Shahzad and Udomene [13], Khan and Takahashi [6], Khan and Ud-din [7] and Xu and Noor [15].

\section{REFERENCES}

[1] S.S. Chang, Some results for asymptotically pseudo-contractive mapping and asymptotically nonexpansive mappings, Proc. Amer. Math. Soc., Vol. 129, No. 3 (2001), 845-853.

[2] S.S. Chang, Iterative approximation problem of fixed points for asymptotically nonexpansive mappings in Banach spaces, Acta Math. Appl., Vol. 24, No. 2 (2001), 236-241.

[3] S.S. Chang, On the approximating problem of fixed points for asymptotically nonexpansive mappings, Indian J. Pure and Appl., Vol. 32, No. 9 (2001), 1-11.

[4] M.K. Ghosh and L. Debnath, Convergence of Ishikawa iterates of quasi-nonexpansive mappings, J. Math. Anal. Appl., Vol. 207 (1997), 96-103.

[5] Hafiz Fukhar-ud-din, Safeer Hussain Khan, Convergence of iterates with errors of asymptotically quasi-nonexpansive mappings and applications, J. Math. Anal. Appl., Vol. 328 (2007), 821-829.

[6] S.H. Khan and W. Takahashi, Approximating common fixed points of two asymptotically nonexpansive mappings, Sci. Math. Jpn., Vol. 53 (2001), 143-148.

[7] S.H. Khan and H. Fukhar-ud-din, Weak and strong convergence of a scheme with errors for two nonexpansive mappings, Nonlinear Analysis, Vol. 61 (2005), 1295-1301.

[8] J.K. Kim, K.H. Kim and K.S. Kim, Convergence theorems of modified three-step iterative sequences with mixed errors for asymptotically quasi-nonexpansive mappings in Banach spaces, PanAmerican Math. Jour., Vol. 14, No, 1 (2004), 45-54.

[9] Q.H. Liu, Iterative sequences for asymptotically quasi-nonexpansive mappings, J. Math. Anal. Appl., Vol. 259 (2001), 1-7.

[10] Q.H. Liu, Iterative sequences for asymptotically quasi-nonexpansive mappings with error member, J. Math. Anal. Appl., Vol. 259 (2001), 18-24.

[11] Q.H. Liu, Iterative sequences for asymptotically quasi-nonexpansive mappings with error member of uniformly convex Banach spaces, J. Math. Anal. Appl., Vol. 266 (2002), 468-471.

[12] W.V. Petryshyn and T.E. Williamson, Strong and weak convergence of the sequence of successive approximations for quasi-nonexpansive mappings, J. Math. Anal. Appl., Vol. 43 (1973), 459-497.

[13] N. Shahzad and A. Udomene, Approximating common fixed points of two asymptotically quasi-nonexpansive mappings in Banach spaces, Fixed Point Theory and Applications, Volume 2006, Article ID 18909, pages 1-10.

[14] W. Takahashi, A convexity in metric space and nonexpansive mappings I, Kodai Math. Sem. Rep., Vol. 22 (1970), 142-149.

[15] B.L. Xu and M.A. Noor, Fixed point iterations for asymptotically nonexpansive mappings in Banach spaces, J. Math. Anal. Appl., Vol. 267, No. 2 (2002), 444-453. 


\section{Gurucharan Singh Saluja}

Department of Mathematics \& Information Technology

Govt. College of Science

RAIPUR-492101(ChHATTISGARH)

INDIA

E-mail address: saluja_1963@rediffmail.com

\section{Hemant Kumar Nashine}

Department of Mathematics

Disha Institute of Management and Technology

Satya Vihar, Vidhansabha - Chandrakhuri Marg

(Baloda Bazar Road), Mandir Hasaud,

RAIPUR-492101(CHHATTISGARH)

INDIA

E-mail address: hnashine@rediffmail.com

hemantnashine@rediffmail.com 